\title{
Early anticoagulation therapy for severe burns complicated by inhalation injury in a rabbit model
}

\author{
ZHONG-HUA FU ${ }^{1}$, GUANG-HUA GUO ${ }^{1}$, ZHEN-FANG XIONG ${ }^{2}$, \\ XINCHENG LIAO $^{1}$, MING-ZHUO LIU ${ }^{1}$ and JINHUA LUO ${ }^{1}$ \\ Departments of ${ }^{1}$ Burns and ${ }^{2}$ Pathology, The First Affiliated Hospital of Nanchang University, \\ Nanchang, Jiangxi 330006, P.R. China
}

Received January 20, 2016; Accepted January 9, 2017

DOI: $10.3892 / \mathrm{mmr} .2017 .7537$

\begin{abstract}
The aim of the present study was to determine the effects of early anticoagulation treatment on severe burns complicated by inhalation injury in a rabbit model. Under anesthetization, an electrical burns instrument $\left(100^{\circ} \mathrm{C}\right)$ was used to scald the backs of rabbits for $15 \mathrm{sec}$, which established a 30\% III severe burns model. Treatment of the rabbits with early anticoagulation effectively improved the severe burns complicated by inhalation injury-induced lung injury, reduced $\mathrm{PaO}_{2}, \mathrm{PaCO}_{2}$ and $\mathrm{SPO}_{2}$ levels, suppressed the expression of tumor necrosis factor- $\alpha$, interleukin (IL)- $1 \beta$ and IL- 6 , and increased the activity of IL-10. In addition, it was found that early anticoagulation treatment effectively suppressed the activities of caspase-3 and caspase-9, upregulated the protein expression of vascular endothelial growth factor (VEGF) and decreased the protein expression of protease-activated receptor 1 (PAR1) in the severe burns model. It was concluded that early anticoagulation treatment affected the severe burns complicated by inhalation injury in a rabbit model through the upregulation of VEGF and downregulation of PAR1 signaling pathways. Thus, early anticoagulation is a potential therapeutic option for severe burns complicated by inhalation injury.
\end{abstract}

\section{Introduction}

Patients with severe burns are a commonly seen clinically and are associated with high mortality rates (1). Burns patients may also suffer from inhalation injury, which can aggravate severity. Patients with severe burns exhibit substantial body trauma with early inflammatory responses $(2,3)$. Clinically, uncontrollable systemic inflammatory response syndromes

Correspondence to: Mr. Guang-Hua Guo, Department of Burns, The First Affiliated Hospital of Nanchang University, 17 YongWai Road, Donghu, Nanchang, Jiangxi 330006, P.R. China E-mail: gqozk545@163.com

Key words: early anticoagulation, severe burns, inhalation injury, vascular endothelial growth factor, protease-activated receptor 1 are readily triggered, which affect system functions and lead to multiple organ failures (4).

In the early stage following a severe burn, effective circulating blood volumes are reduced and blood is concentrated (5). Thus, the blood clotting response is induced. The hypercoagulability of blood lowers and inhibits blood flow, and affects the speed of fluid resuscitation speed, resulting in tissue damage as the ischemic conditions of blood capillaries cannot be quickly and effectively corrected (6). In addition, the hypercoagulability of blood inhibits the percolatation of pulmonary alveoli, inhibits pulmonary alveoli, reduces areas of blood gas exchange, decreases the ratio of ventilation/blood flow and affects oxygenation (7). When hypercoagulability occurs, numerous blood platelets are consumed, which causes secondary hyperfibrinolysis. In addition, substantial transfusion to combat shock in the early stage is likely to result in bleeding tendency and affect wound repair (8). Patients with severe burns may exhibit diffuse vascular hemorrhage, and it has been suggested that a decline in the population of blood platelets is associated with the age and areas of the burns. The prognosis of patients can be predicted by monitoring blood platelet volumes (5). Therefore, the present study aimed to determine whether organ functions can be improved and whether the rapid decline in blood platelet volumes can be inhibited in cases of serious burns through the application of an anticoagulant to improve hypercoagulability (7).

Investigations have examined the combination of inhalation injury in severe burns, and aerosol inhalation of heparin at an early stage can effectively improve coagulation disorders of the lungs, reduce cellular infiltration, hyperemia and edema, improve lung injury and inhibit the progression to acute respiratory distress syndromes $(6,9)$. Similarly, the application of other anticoagulants, including antithrombase, can alleviate lung injuries in animals with severe burns and inhalation injury, and can relieve pulmonary functions (10). Clinical trials have shown that the inhalation of heparin can ease pulmonary inflammation following inhalation injury and decrease mechanical ventilation time, however, this remains controversial (11). It was previously found that the use of heparin for inhalation injury provided no advantages in improving other syndromes, with the exception of increasing oxygen partial pressures (12). In addition, the application of heparin may lead to a tendency to bleed, active hemorrhage and peptic ulcers. 
However, aerosol inhalation of heparin in patients with acute lung injury caused by inhalation injury is improved without obvious hemorrhagic tendency (13). The present study focused in the effects of the use of early anticoagulation therapy on a rabbit model of severe burns complicated by inhalation injury.

\section{Materials and methods}

Establishment and grouping of animal models. Male adult New Zealand rabbits (2.5 $\pm 1 \mathrm{~kg}$, aged 4-5 months) were purchased from Laboratory Animal Center of Nanchang University (Nanchang, China) and housed at $23-24^{\circ} \mathrm{C}, 8: 00$ a.m. to 8:00 p.m. lighting, with access to food/water, and were randomly allocated into five groups: Sham group; severe burns model group; early heparin treatment group; early antithrombin group; early heparin + antithrombin group ( $\mathrm{n}=8$ in each group). Sodium sulphide $(10 \% \mathrm{v} / \mathrm{v})$ was used to depilate the back of each rabbit and pentobarbital sodium $(40 \mathrm{mg} / \mathrm{kg}$ ) was then injected into the auricular vein. Under anesthetization, an electrical burns instrument $\left(100^{\circ} \mathrm{C}\right)$ was used to scald at the backs of the rabbits for $15 \mathrm{sec}$, which established a 30\% III severe burns model. The scald area was calculated as follows: Area of severe burn/total area. After $1 \mathrm{~h}$, the rabbits in the sham and severe burns model were injected with $100 \mu 1$ normal saline into the auricular vein. The rabbits in the early heparin treatment group, early antithrombin group and early heparin + antithrombin group were injected with heparin $(1,000 \mathrm{U} / \mathrm{kg})$, antithrombin $(25 \mathrm{U} / \mathrm{kg})$ or heparin $(1,000 \mathrm{U} / \mathrm{kg})+$ antithrombin $(25 \mathrm{U} / \mathrm{kg})$, respectively, into the auricular vein. The study was approved by the ethics committee of The First Affiliated Hospital of Nanchang University.

Histology. Rabbits were sacrificed with abundant pentobarbital after treatment, and lung tissue samples were harvested and immersed in $10 \%$ (v/v) formalin for $48 \mathrm{~h}$. The tissue samples were exposed to xylene with dewaxing for $10 \mathrm{~min}$ and dehydrated using different concentrations of alcohol. The samples were then cut into $4 \mu \mathrm{M}$ sections, stained with hematoxylin and eosin and observed under light microscopy (BH3-MJL; Olympus, Tokyo, Japan).

Biological indicators. At 0,2, 8, 12 and 24 h post-treatment, measurements of $\mathrm{PaO}_{2}, \mathrm{PaCO}_{2}$, platelet counts, blood coagulation function, heart rate and cardiac output were obtained using SLE5000 high-frequency oscillatory ventilators (SLE, Co., Croydon, UK), an ABL blood gas analyzer (Radiometer, Copenhagen, Denmark), measuring respiratory pressure with a gas flow rate meter (5800, Hudson RCI; Temecula, CA, USA) and cardiac output apparatus (N600X, Nellcor; Covidien, Boulder, CO, USA).

ELISA measurements. At 0, 2, 8, 12 and 24 h post-treatment, venous blood was collected and centrifuged at 8,000 x $\mathrm{g}$ for $10 \mathrm{~min}$ at $4^{\circ} \mathrm{C}$. According to the manufacturer's protocol, the contents of tumor necrosis factor (TNF)- $\alpha$, interleukin (IL)-1 $\beta$, IL-6 and IL-10 were measured using an automated microplate reader (Bioelisa ELx800 ${ }^{\mathrm{TM}}$ absorbance reader; BioTek Instruments, Inc., Winooski, VT, USA).

Western blot analysis. At 0, 2, 8, 12 and $24 \mathrm{~h}$ post-treatment, the burn tissue samples were collected and homogenized, and centrifuged at $8,000 \mathrm{x}$ g for $15 \mathrm{~min}$ at $4^{\circ} \mathrm{C}$. The supernatant was used to measure total protein concentrations using a BCA kit. The proteins (50 $\mu \mathrm{g})$ were separated using $10 \%$ SDS polyacrylamide gel electrophoresis and transferred onto a nitrocellulose membrane. The membrane was blocked with $5 \%$ milk in TBST for $1 \mathrm{~h}$ at $4^{\circ} \mathrm{C}$ and incubated with the following antibodies: NF-кB/p65 (cat. no. sc-109, 1:2,000; Santa Cruz Biotechnology, Inc., Franklin Lakes, NJ, USA), vascular endothelial growth factor (VEGF; cat. no. sc-13083, 1:4,000; Santa Cruz Biotechnology, Inc.), protease-activated receptor 1 (PAR1; cat. no. SAB5300042, 1:3,000; Sigma-Aldrich; Merck KGaA, Darmstadt, Germany) and $\beta$-actin (cat. no. sc-7210, 1:5,000; Santa Cruz Biotechnology, Inc.) at $4^{\circ} \mathrm{C}$ overnight. Subsequently, the membrane was incubated with sheep anti-rabbit or mouse IgG (cat nos. 14708 or 14709, 1:1,000; Cell Signaling Technology, Inc.) at room temperature for $2 \mathrm{~h}$, and bands were revealed using an ECL kit (Cell Signaling Technology, Inc., Danvers, MA, USA) and quantified using Quantity One software version 3.0 (Bio-Rad, Hercules, CA, USA).

Measuring caspase-3 and caspase-9 activities. At 0, 2, 8, 12 and $24 \mathrm{~h}$ post-treatment, the burn tissue samples were collected and homogenized using radioimmunoprecipitation assay buffer, and centrifuged at $8,000 \mathrm{x} \mathrm{g}$ at $4^{\circ} \mathrm{C}$ for $15 \mathrm{~min}$. The supernatant was used to measure total protein concentrations using a BCA kit. The proteins $(50 \mu \mathrm{g})$ were incubated with Ac-DEVD-pNA (caspase-3, 2 mM) or Ac-LEHD-pNA (caspase-9, $2 \mathrm{mM}$ ) at $37^{\circ} \mathrm{C}$ for $1 \mathrm{~h}$, following which the activity of caspase-3 was measured using an automated microplate reader (Bioelisa ELx800 ${ }^{\mathrm{TM}}$ Absorbance Reader; BioTek Instruments, Inc.) at $405 \mathrm{~nm}$.

Statistical analysis. All data are expressed as the mean \pm standard deviation. One-way analysis of variance and pairwise comparisons were performed using SPSS software version 17.0 (SPSS, Inc., Chicago, IL, USA). P<0.05 was considered to indicate a statistically significant difference.

\section{Results}

Effect of early anticoagulation treatment on lung injury in a rabbit model of severe burns complicated by inhalation injury. As shown in Fig. 1, lung injury in the severe burns complicated by inhalation injury model group was higher, compared with that in the sham group. Treatment with either heparin or antithrombin effectively inhibited the severe burns complicated by inhalation injury-induced lung injury in rabbits, compared with the model group (Fig. 1). Treatment with early heparin + antithrombin significantly inhibited the severe burns complicated by inhalation injury-induced lung injury in rabbits, compared with that in the heparin treatment group (Fig. 1)

Effect of early anticoagulation treatment on the levels of $\mathrm{PaO}_{2}$ and $\mathrm{PaCO}_{2}$ in a rabbit model of severe burns complicated by inhalation injury. The levels of $\mathrm{PaO}_{2}$ (Fig. 2A) and $\mathrm{PaCO}_{2}$ (Fig. 2B) in the severe burns complicated by inhalation injury-induced lung injury group were higher, compared with those of the control sham group. Treatment with either heparin 


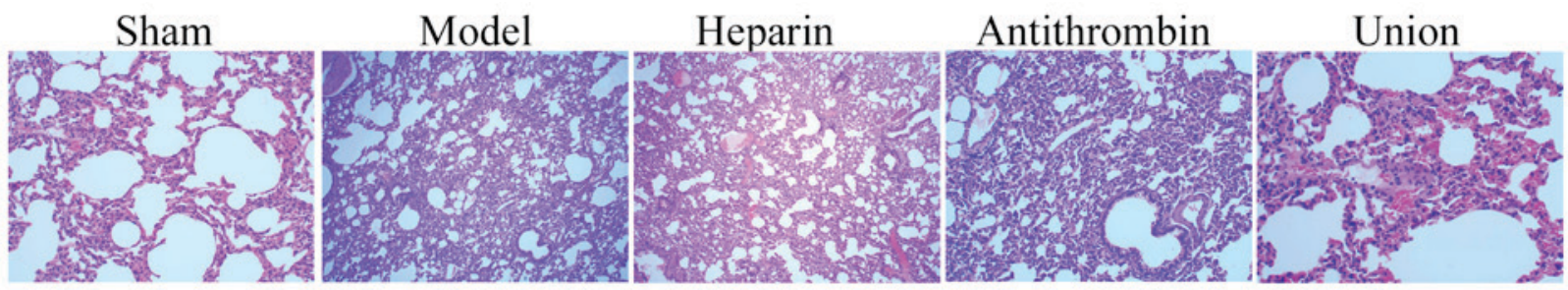

Figure 1. Effect of early anticoagulation therapy on lung injury in a rabbit model of severe burns complicated by inhalation injury. Magnification, x10. Sham, sham group; model, severe burns model group; heparin, early heparin treatment group; antithrombin, early antithrombin group; union, early heparin + antithrombin group.

A

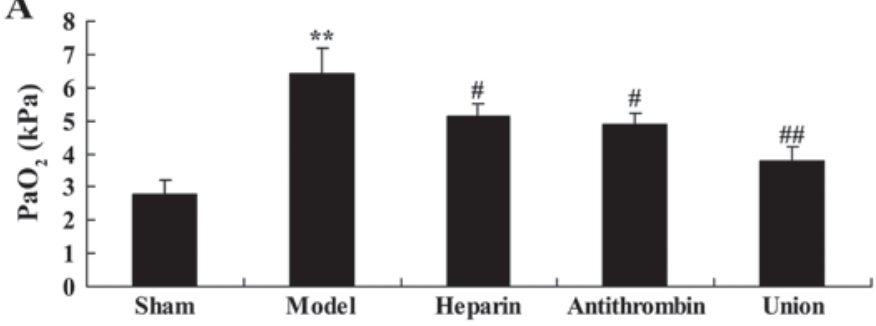

B

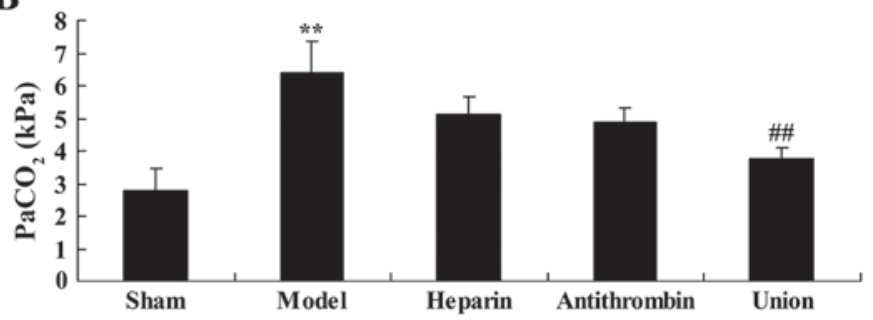

Figure 2. Effect of early anticoagulation therapy on the levels of $\mathrm{PaO}_{2}$ and $\mathrm{PaCO}_{2}$ in a rabbit model of severe burns complicated by inhalation injury. Early anticoagulation therapy on the levels of (A) $\mathrm{PaO}_{2}$ and (B) $\mathrm{PaCO}_{2}$ in a rabbit model of severe burns complicated inhalation injury. Sham, sham group; model, severe burns model group; heparin, early heparin treatment group; antithrombin, early antithrombin group; union, early heparin + antithrombin group. ${ }^{* *} \mathrm{P}<0.01$, compared with the sham group; ${ }^{\#} \mathrm{P}<0.01$, compared with the model group; ${ }^{\# \#} \mathrm{P}<0.01$, compared with the heparin treatment group.

or antithrombin significantly reduced the level of severe burns complicated by inhalation injury-induced $\mathrm{PaO}_{2}$ in the rabbits, compared with that in the model group (Fig. 2). However, early heparin + antithrombin treatment effectively reduced the levels of $\mathrm{PaO}_{2}$ and $\mathrm{PaCO}_{2}$ in the rabbits, compared with those in the heparin treatment group (Fig. 2).

Effect of early anticoagulation treatment on heart rate in a rabbit model of severe burns complicated by inhalation injury. Compared with the sham group, heart rate was effectively weakened in the severe burns complicated by inhalation injury rabbit model. As shown in Fig. 3, treatment with either heparin or antithrombin effectively weakened the severe burns complicated by inhalation injury-induced heart rate in rabbits, compared with that in the model group. Early heparin + antithrombin treatment significantly weakened the severe burns complicated by inhalation injury-induced heart rate in the rabbits, compared with that in the heparin treatment group (Fig. 3).

Effect of early anticoagulation treatment on $\mathrm{SPO}_{2}$ and central venous pressure $(C V P)$ in a rabbit model of severe burns complicated by inhalation injury. After $1 \mathrm{~h}$, the level of $\mathrm{SPO}_{2}$ in the rabbit model of severe burns complicated by inhalation injury were markedly higher, compared with that of the sham group (Fig. 4A). Treatment with either heparin or antithrombin effectively inhibited the severe burns complicated by inhalation injury-induced level of $\mathrm{SPO}_{2}$ in the rabbits, compared with that in the model group (Fig. 4A). Of note, early heparin + antithrombin treatment significantly inhibited induction of $\mathrm{SPO}_{2}$, compared with that in the heparin treatment group (Fig. 4A). However, no statistically significant differences in CVP were observed between the sham, model, heparin, antithrombin and early heparin + antithrombin groups (Fig. 4B).

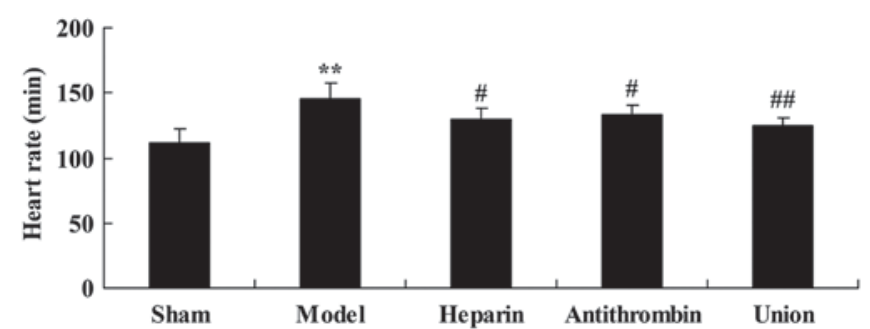

Figure 3. Effect of early anticoagulation therapy on the heart rate of rabbits in a model of severe burns complicated by inhalation injury. Sham, sham group; model, severe burns model group; heparin, early heparin treatment group; antithrombin, early antithrombin group; union, early heparin + antithrombin group. ${ }^{* *} \mathrm{P}<0.01$, compared with the sham group; ${ }^{\#} \mathrm{P}<0.01$, compared with the model group; ${ }^{\# \#} \mathrm{P}<0.01$, compared with the heparin treatment group.

Effect of early anticoagulation treatment on levels of TNF- $\alpha$, $I L-1 \beta, I L-6$ and IL-10 in a rabbit model of severe burns complicated by inhalation injury. There were significant increases in the levels of TNF- $\alpha$, IL-1 $\beta$ and IL-6, and inhibition of IL-10 in the severe burns complicated by inhalation injury model group, compared with levels in the sham group (Fig. 5). Pretreatment with either heparin or antithrombin effectively reversed the induction of inflammation in the rabbits, compared with that in the model group (Fig. 5). Early heparin + antithrombin treatment significantly inhibited inflammation in the rabbits, compared with that in the heparin treatment group (Fig. 5).

Effect of early anticoagulation treatment on activities of caspase-3/caspase-9 in a rabbit model of severe burns complicated by inhalation injury. The present study found that caspase- 3 and caspase- 9 were activated in the rabbit model of severe burns complicated by inhalation injury, compared with 
A

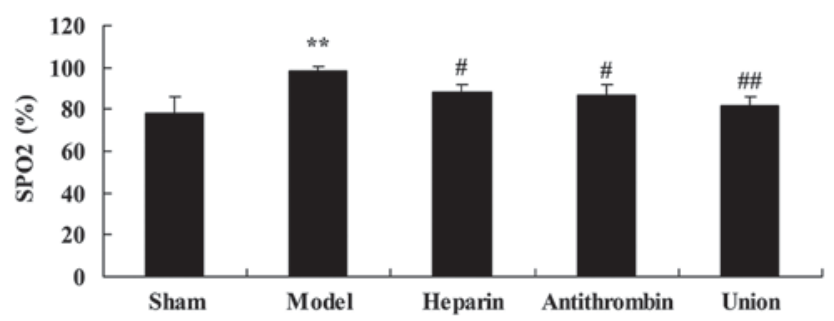

B

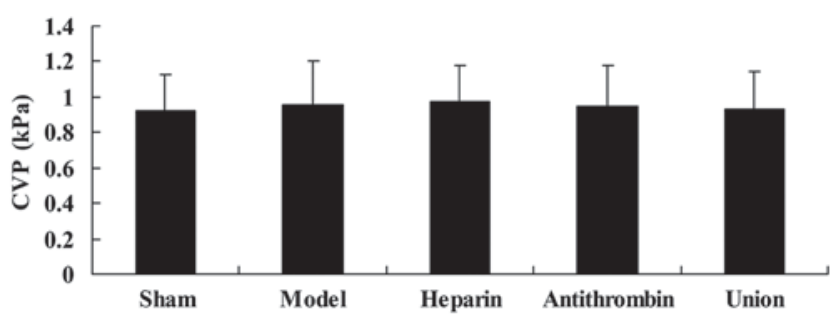

Figure 4. Effect of early anticoagulation therapy on $\mathrm{SPO}_{2}$ and CVP in a rabbit model of severe burns complicated by inhalation injury. Effects of early anticoagulation therapy on (A) $\mathrm{SPO}_{2}$ and (B) CVP in a rabbit model of severe burns complicated inhalation injury. Sham, sham group; model, severe burns model group; heparin, early heparin treatment group; antithrombin, early antithrombin group; union, early heparin + antithrombin group. ${ }^{* *} \mathrm{P}<0.01$, compared with sham group; ${ }^{\#} \mathrm{P}<0.01$, compared with the model group; ${ }^{\# \#} \mathrm{P}<0.01$, compared with the heparin treatment group. CVP, central venous pressure.
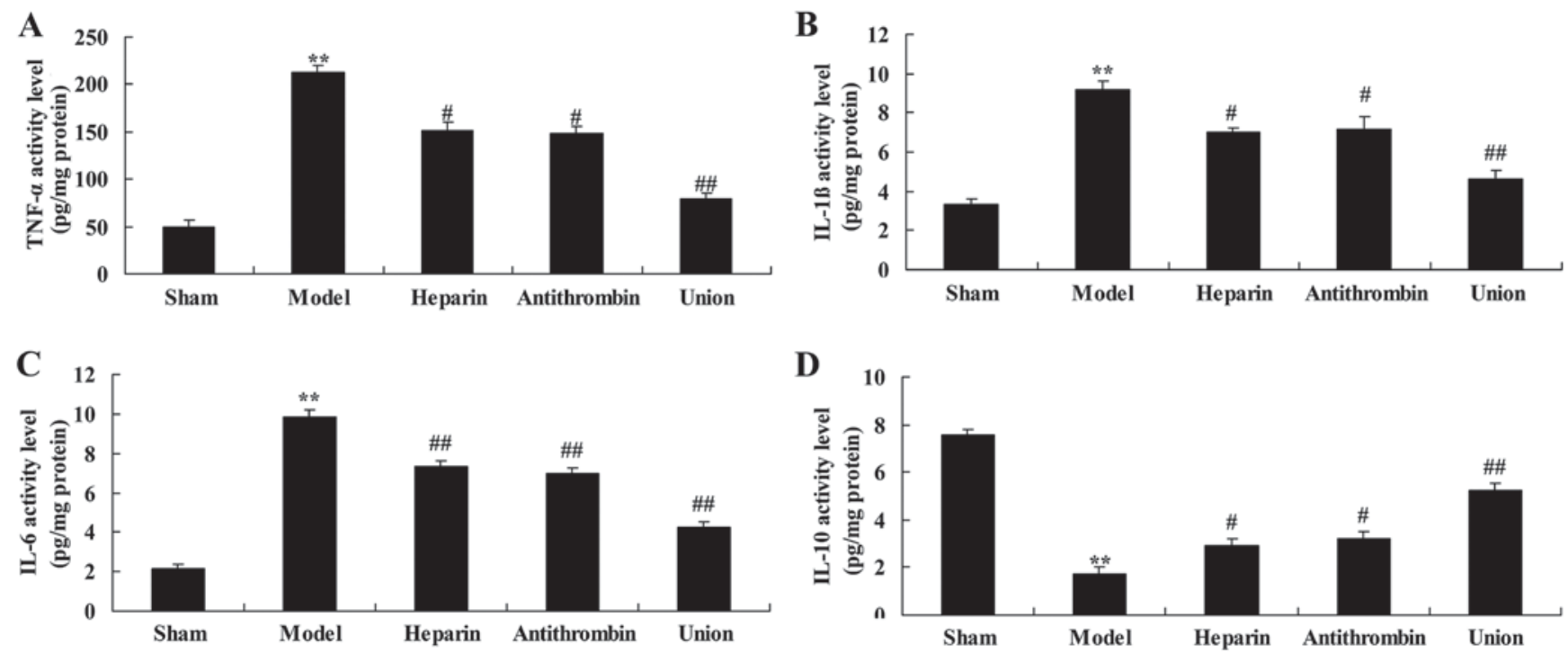

Figure 5. Effect of early anticoagulation therapy on TNF- $\alpha$, IL-1 $\beta$, IL-6 and IL-10 content in a rabbit model of severe burns complicated by inhalation injury. Effects of early anticoagulation therapy on levels of (A) TNF- $\alpha$, (B) IL-1 $\beta$, (C) IL-6 and (D) IL-10 in a rabbit model of severe burns complicated inhalation injury. Sham, sham group; model, severe burns model group; heparin, early heparin treatment group; antithrombin, early antithrombin group; union, early heparin + antithrombin group. ${ }^{* *} \mathrm{P}<0.01$, compared with the sham group; ${ }^{\#} \mathrm{P}<0.01$, compared with the model group; ${ }^{\# \prime} \mathrm{P}<0.01$, compared with the heparin treatment group. TNF- $\alpha$, tumor necrosis factor- $\alpha$; IL, interleukin.

A

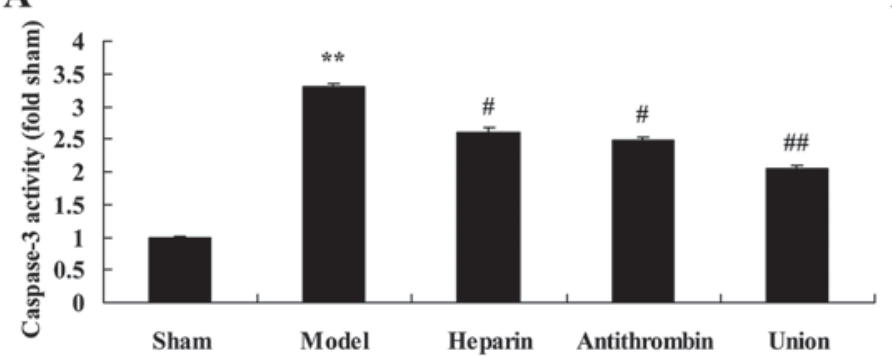

B

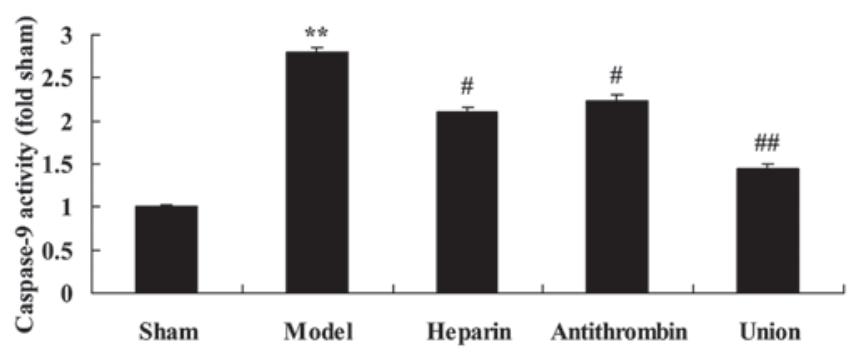

Figure 6. Effects of early anticoagulation therapy on the levels of caspase- 3 and caspase- 9 in a rabbit model of severe burns complicated by inhalation injury. Effects of early anticoagulation therapy on the levels of (A) caspase-3 and (B) caspase-9 in a rabbit model of severe burns complicated inhalation injury. Sham, sham group; model, severe burns model group; heparin, early heparin treatment group; antithrombin, early antithrombin group; union, early heparin + antithrombin group. ${ }^{* *} \mathrm{P}<0.01$, compared with the heparin treatment group.

the sham group (Fig. 6A and B). Treatment with either heparin or antithrombin effectively inhibited the activities of caspase-3 and caspase- 9 in the rabbits, compared with those in the model group (Fig. 6). However, early heparin + antithrombin treatment significantly suppressed the activities of caspase- 3 and caspase- 9 in the rabbits, compared with those in the heparin treatment group (Fig. 6).

Effect of early anticoagulation therapy on the protein expression of $\mathrm{NF}-\kappa \mathrm{B} / \mathrm{p} 65$ in a rabbit model of severe burns 
A

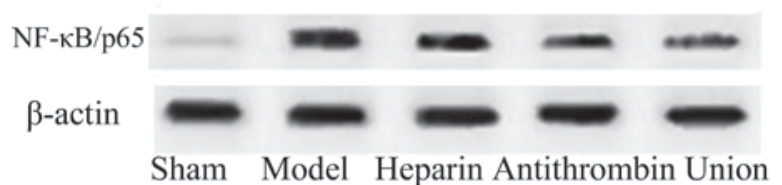

B

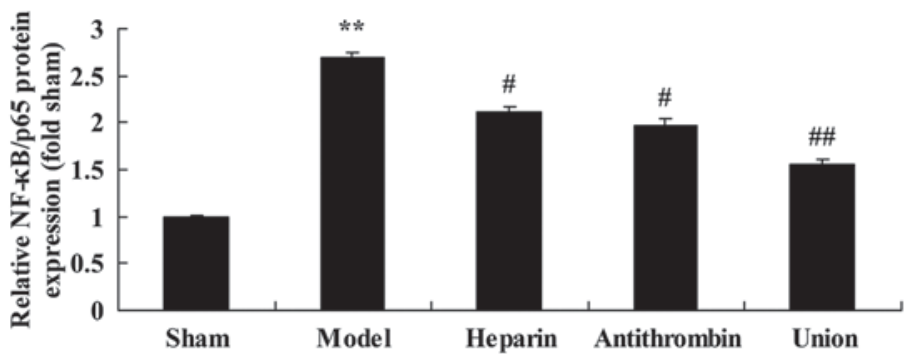

Figure 7. Effects of early anticoagulation therapy on the protein expression of NF- $\kappa \mathrm{B} / \mathrm{p} 65$ in a rabbit model of severe burns complicated by inhalation injury. (A) Effects of early anticoagulation on the protein expression of NF- $\kappa \mathrm{B} / \mathrm{p} 65$ were determined using western blot analysis. (B) Statistical analysis of the protein expression of $\mathrm{NF}-\kappa \mathrm{B} / \mathrm{p} 65$. Sham, sham group; model, severe burns model group; heparin, early heparin treatment group; antithrombin, early antithrombin group; union, early heparin + antithrombin group. ${ }^{* *} \mathrm{P}<0.01$, compared with the sham group; ${ }^{\#} \mathrm{P}<0.01$, compared with the model group; ${ }^{\# \#} \mathrm{P}<0.01$, compared with the heparin treatment group. $\mathrm{NF}-\kappa \mathrm{B}$, nuclear factor- $\kappa \mathrm{B}$.

A

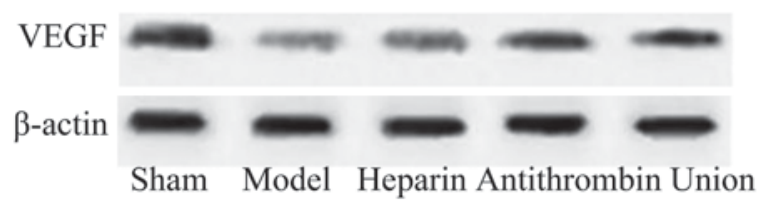

B

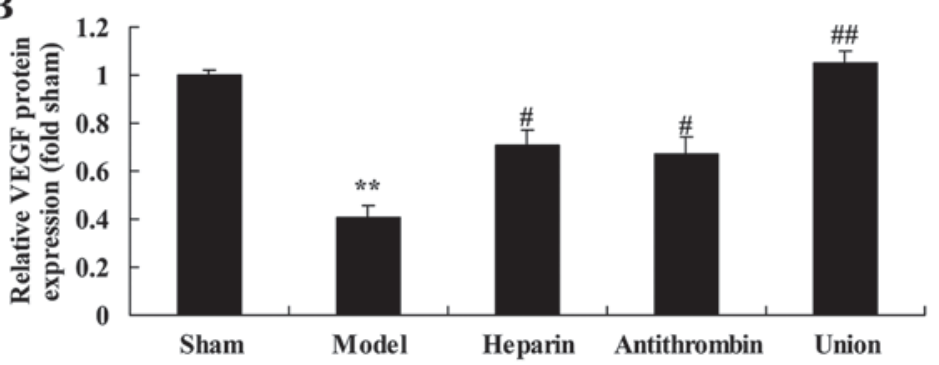

Figure 8. Effects of early anticoagulation on the protein expression of VEGF in a rabbit model of severe burns complicated by inhalation injury. (A) Effects of early anticoagulation on the protein expression of VEGF were determined using western blot analysis. (B) Statistical analysis of the protein expression of VEGF. Sham, sham group; model, severe burns model group; heparin, early heparin treatment group; antithrombin, early antithrombin group; union, early heparin + antithrombin group. ${ }^{* *} \mathrm{P}<0.01$, compared with the sham group; ${ }^{\#} \mathrm{P}<0.01$, compared with the model group; ${ }^{\# \#} \mathrm{P}<0.01$, compared with the heparin treatment group. VEGF, vascular endothelial growth factor.
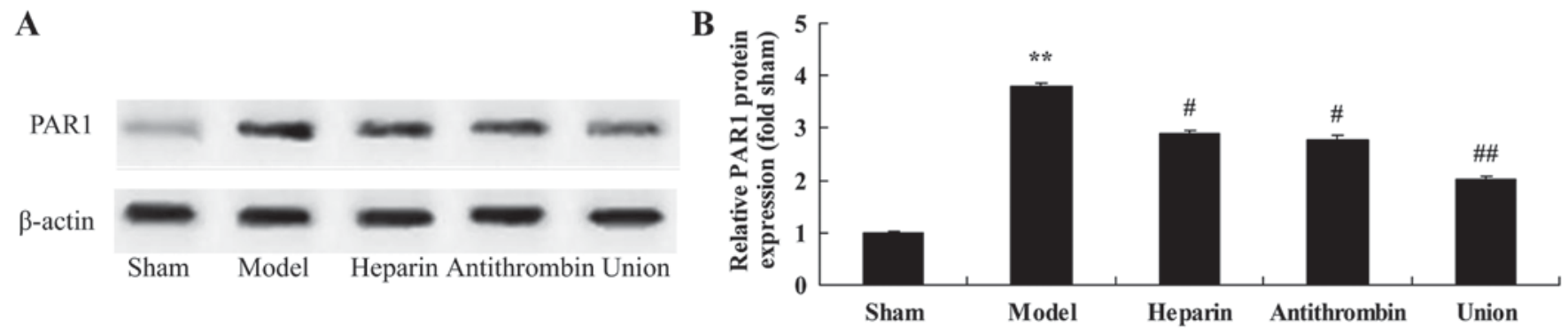

Figure 9. Effects of early anticoagulation therapy on the protein expression of PAR1 in a rabbit model of severe burns complicated by inhalation injury. (A) Effects of early anticoagulation therapy on the protein expression of PAR1 were determined using western blot analysis. (B) Statistical analysis of the protein expression of PAR1. Sham, sham group; model, severe burns model group; heparin, early heparin treatment group; antithrombin, early antithrombin group; union, early heparin + antithrombin group. ${ }^{* *} \mathrm{P}<0.01$, compared with the sham group; ${ }^{\#} \mathrm{P}<0.01$, compared with the model group; ${ }^{\# \#} \mathrm{P}<0.01$, compared with the heparin treatment group. PAR1, protease-activated receptor 1.

complicated by inhalation injury. As shown in Fig. 7A, the protein expression of $\mathrm{NF}-\kappa \mathrm{B} / \mathrm{p} 65$ was enhanced in the severe burns complicated by inhalation injury model group, compared with the sham group. Treatment with either heparin or antithrombin effectively suppressed the protein expression of $\mathrm{NF}-\kappa \mathrm{B} / \mathrm{p} 65$ in the rabbits, compared with the model group (Fig. 7B). Treatment with early heparin + antithrombin significantly suppressed the protein expression of $\mathrm{NF}-\kappa \mathrm{B} / \mathrm{p} 65$ in the rabbits, compared with the heparin treatment group (Fig. 7B).

Effect of early anticoagulation therapy on the protein expression of VEGF in a rabbit model of severe burns complicated by inhalation injury. The protein expression of VEGF was inhibited in the model group, compared with that in the sham group (Fig. 8A). Compared with the model group, treatment with either heparin or antithrombin effectively increased the protein expression of VEGF in the rabbit, compared with that in the model group (Fig. 8B). Treatment with early heparin + antithrombin significantly increased the protein expression of VEGF in the rabbits, compared with that in the heparin treatment group (Fig. 8B).

Effect of early anticoagulation therapy on the protein expression of PARI in a rabbit model of severe burns complicated 
by inhalation injury. Compared with the rabbits in the sham group, severe burns complicated by inhalation injury effectively promoted the protein expression of PAR 1 in the rabbit model (Fig. 9A). Treatment with either heparin or antithrombin effectively suppressed the protein expression of PAR1 in the rabbits, compared with that in the model group (Fig. 9B). However, early heparin + antithrombin significantly suppressed the protein expression of PAR1 in the rabbits, compared with that in the heparin treatment group (Fig. 9B).

\section{Discussion}

Inhalation injury is an important cause of injury in burns patients. The mortality rate for patients with severe burns is $8 \%$ in China (3). Body surface burns and inhalation injury have cumulative effects. It has been reported that the occurrence rate of respiratory failure solely caused by inhalation injury is only $1 \%$, whereas this rate increases to $6 \%$ when inhalation injury is present in combination with body surface burns (2). Therefore, it is vital to increase recovery rates in burns patients with concomitant severe inhalation injury. Following a severe burn, compensatory reactions lead to shrinkage of the gastrointestinal digestive tract mucosa, reduction in blood flow volume, decreased secretion of gastric mucoid and ischemia reperfusion injury (14). Thus, the digestive tract mucosa is injured. Whether the application of anticoagulants at an early stage can protect the digestive tract mucosa and reduce the gastrointestinal reaction remains to be elucidated (15). In the present study, it was demonstrated that early heparin + antithrombin treatment significantly inhibited the lung injury induced by severe burns complicated by inhalation injury in rabbits, and reduced the levels of $\mathrm{PaO}_{2}, \mathrm{PaCO}_{2}$ and $\mathrm{SPO}_{2}$.

Between 4 and $24 \mathrm{~h}$ following the occurrence of a severe burn, myocardial contractility is reduced and the leakage of sarcoplasmic reticulum results in myocardial dysfunction (8). It has been reported that, following a severe burn, autophagy of the myocardium is enhanced and the numbers of dead cells are increased, which may be one cause of myocardial function injury (5). Reports on the effects of anticoagulant therapy in the early stage on myocardial function are limited (6). Individual animal experiments have found that the intravenous injection of antithrombase can modify myocardia and alleviate hyposarca of burns complicated by inhalation injury (6). In the present study, early heparin + antithrombin treatment significantly inhibited inflammation and suppressed the activities of caspase-3 and caspase- 9 through the inhibition of $\mathrm{NF}-\kappa \mathrm{B} / \mathrm{p} 65$ in the rabbit model of severe burns complicated by inhalation injury. Levy et al (16) also showed that antithrombin possesses noteworthy anti-inflammatory properties.

Inhalation injury, ischemia and hemopoietin can induce the chemical chemotactic activity of VEGF and accumulate monocytes (17). By increasing vascular permeability, VEGF can ease the exudation of plasma proteins, including albumin and cellulose (18). The results of the present study confirmed that treatment with early heparin + antithrombin significantly activated the protein expression of VEGF in the rabbit model of severe burns complicated by inhalation injury. Azhar et al (19) reported that antithrombin has an antiangiogenic function through conformational variation.
The mRNA and proteins expression levels of PAR-1 in unstable plaques are increased, indicating that PAR-1 is a promoting factor for unstable plaques (20). According to previous studies, thrombin exerts its pro-inflammatory and blood vessel-regulating functions through several pathways. Firstly, it promotes the expression of cytokines (IL-6 and IL-8) and is involved in the inflammatory response (21). Secondly, it promotes the expression of monocyte chemoattractant protein-1 and increases that of vascular cell adhesion molecule 1 (22). Therefore, if the expression of PAR-1 is inhibited, injury by thrombin to the blood vessels is reduced.

PAR-1 is the primary receptor of thrombin, which is a multi-functional protease. In previous years, studies on thrombin have been expanded to its non-anticoagulation functions, which have introduced a novel direction for investigations on atherosclerosis (23). PAR-1 can be expressed on blood platelets and other tissues, including vascular endothelial cells, vascular smooth muscle cells and brain tissues (20). The occurrence rates of atherosclerosis in rats with PAR-1 gene-knockout are low, indicating that PAR-1 is essential in anti-atherosclerosis investigations (24). However, the results of the present study showed that early heparin + antithrombin treatment significantly suppressed the protein expression of PAR1 in the rabbit model of severe burns complicated by inhalation injury. Balzarotti et al (25) confirmed that low molecular weight heparin decreases cell growth and cell invasion in primary cell cultures of high-grade gliomas through the expression of VEGF and PAR1.

In conclusion, the results of the present study demonstrated that early anticoagulation therapy inhibited the lung injury induced by severe burns complicated by inhalation injury, reduced levels of $\mathrm{PaO}_{2}, \mathrm{PaCO}_{2}$ and $\mathrm{SPO}_{2}$, was involved in the anti-inflammatory effect, and adjusted the levels of VEGF and PAR-1 in the rabbit model of severe burns complicated by inhalation injury. Thus, early anticoagulation treatment is a potential therapeutic option for the treatment of severe burns complicated by inhalation injury.

\section{References}

1. Zhang Y, Cai B, Jiang H, Yan H, Yang H, Peng J, Wang W, Ma S, Wu X and Peng X: Use of $1 \mathrm{H}$-nuclear magnetic resonance to screen a set of biomarkers for monitoring metabolic disturbances in severe burn patients. Crit Care 18: R159, 2014.

2. Jeschke MG, Gauglitz GG, Kulp GA, Finnerty CC, Williams FN, Kraft R, Suman OE, Mlcak RP and Herndon DN: Long-term persistance of the pathophysiologic response to severe burn injury. PLoS One 6: e21245, 2011.

3. Gray P, Kirby J, Smith MT, Cabot PJ, Williams B, Doecke J and Cramond T: Pregabalin in severe burn injury pain: A double-blind, randomised placebo-controlled trial. Pain 152: 1279-1288, 2011.

4. Diaz EC, Herndon DN, Lee J, Porter C, Cotter M, Suman OE, Sidossis LS and Børsheim E: Predictors of muscle protein synthesis after severe pediatric burns. J Trauma Acute Care Surg 78: 816-822, 2015.

5. Mariano F, Tedeschi L, Morselli M, Stella M and Triolo G: Normal citratemia and metabolic tolerance of citrate anticoagulation for hemodiafiltration in severe septic shock burn patients. Intensive Care Med 36: 1735-1743, 2010.

6. Miller AC, Elamin EM and Suffredini AF: Inhaled anticoagulation regimens for the treatment of smoke inhalation-associated acute lung injury: A systematic review. Crit Care Med 42: 413-419, 2014.

7. Gille J, Sablotzki A, Malcharek M, Raff T, Mogk M and Parentin T: Regional citrate anticoagulation for continuous renal replacement therapy in severe burns-a retrospective analysis of a protocol-guided approach. Burns 40: 1593-1601, 2014. 
8. Ono I, Ohura T, Azami K, Hoshi $M$ and Hasegawa $\mathrm{T}$ : Anticoagulation therapy for renal insufficiency after burns. Burns Incl Therm Inj 11: 104-110, 1984.

9. Sorg H, Hoffmann JO, Hoffmann JN and Vollmar B: Analysis of the influence of antithrombin on microvascular thrombosis: Anti-inflammation is crucial for anticoagulation. Intensive Care Med Exp 3: 58, 2015.

10. Mohebali J, Ibrahim AE, MacGillivray TE, Goverman J and Fagan SP: Mitral valve repair via right thoracotomy for multidrug resistant pseudomonal endocarditis in a burn patient: Case report and review of the literature. Burns 41: e47-e50, 2015.

11. PourafkariL, GhaffariS, KhakiN,Hobika GH,Masnadi-ShiraziK and Nader ND: Predictors of hospital mortality and serious complications in patients admitted with excessive warfarin anticoagulation. Thromb Res 137: 79-84, 2016.

12. van den Bergh WM, Lansink-Hartgring AO, van Duijn AL, Engström AE, Lahpor JR and Slooter AJ: Thromboembolic stroke in patients with a HeartMate-II left ventricular assist device-the role of anticoagulation. J Cardiothorac Surg 10: 128, 2015.

13. Moeinipour A, Zarifian A, Sheikh Andalibi MS, Shamloo AS, Ahmadabadi A, Amouzeshi A and Hoseinikhah H: The best anticoagulation therapy in multiple-trauma patients with mechanical heart valves: Evaluation of latest guidelines and studies. Heart Surg Forum 18: E271-E274, 2015.

14. Maybauer MO, Maybauer DM, Fraser JF, Westphal M, Szabó C, Cox RA, Hawkins HK, Traber LD and Traber DL: Combined recombinant human activated protein $\mathrm{C}$ and ceftazidime prevent the onset of acute respiratory distress syndrome in severe sepsis Shock 37: 170-176, 2012.

15. Schleich AR, Schweiger H, Becsey A and Cruse CW: Survival after severe intrathoracic electrical injury. Burns 36: e61-e64, 2010.

16. Levy JH, Sniecinski RM, Welsby IJ and Levi M: Antithrombin: Anti-inflammatory properties and clinical applications. Thromb Haemost 115: 712-728, 2016.
17. Ruan Q,Zhao C,YeZ,Ruan J,Xie Q and Xie W: Effect and possible mechanism of monocyte-derived VEGF on monocyte-endothelial cellular adhesion after electrical burns. Burns 41: 825-832, 2015.

18. Kim YR and Hong SH: Association between the polymorphisms of the vascular endothelial growth factor gene and metabolic syndrome. Biomed Rep 3: 319-326, 2015.

19. Azhar A, Singh P, Rashid Q, Naseem A, Khan MS and Jairajpuri MA: Antiangiogenic function of antithrombin is dependent on its conformational variation: Implication for other serpins. Protein Pept Lett 20: 403-411, 2013.

20. Gurbel PA, Bliden KP, Turner SE, Tantry US, Gesheff MG, Barr TP, Covic L and Kuliopulos A: Cell-penetrating pepducin therapy targeting PAR1 in subjects with coronary artery disease. Arterioscler Thromb Vasc Biol 36: 189-197, 2016.

21. Simmons S, Lee RV, Möller T and Weinstein JR: Thrombin induces release of proinflammatory chemokines interleukin- 8 and interferon- $\gamma$-induced protein-10 from cultured human fetal astrocytes. Neuroreport 24: 36-40, 2013.

22. Tantivejkul K, Loberg RD, Mawocha SC, Day LL, John LS, Pienta BA, Rubin MA and Pienta KJ: PAR1-mediated NFkappaB activation promotes survival of prostate cancer cells through a Bcl-xL-dependent mechanism. J Cell Biochem 96: 641-652, 2005.

23. Hashemzadeh M, Arreguin JM, Roberts T and Movahed MR: A novel inhibitor of protease-activated receptor 1: A review of chemical structure and mode of action. Rev Cardiovasc Med 16: 68-73, 2015.

24. Austin KM, Covic L and Kuliopulos A: Matrix metalloproteases and PAR1 activation. Blood 121: 431-439, 2013.

25. Balzarotti M, Fontana F, Marras C, Boiardi A, Croci D, Ciusani E and Salmaggi A: In vitro study of low molecular weight heparin effect on cell growth and cell invasion in primary cell cultures of high-grade gliomas. Oncol Res 16: 245-250, 2006. 